\title{
Development of a New Adaptive Beam-Forming Technique for Smart Antenna System
}

\author{
Mom Joseph \\ Department of Electrical and \\ Electronics Engineering \\ Federal University of Agriculture \\ Makurdi, Benue State, Nigeria
}

\author{
Olanipekun Tobi \\ Department of Electrical and \\ Electronics Engineering \\ Federal University of Agriculture \\ Makurdi, Benue State, Nigeria
}

\author{
Gabriel A. Igwue \\ Department of Electrical and \\ Electronics Engineering \\ Federal University of Agriculture \\ Makurdi, Benue State, Nigeria
}

\begin{abstract}
Smart antenna is one of the most efficient innovations for maximum interference mitigation, improved tracking of signal of interest and coverage. A systematic implementation of a new beam-forming algorithm technique for smart antenna system has been developed which entails combining linear random antenna configuration with fast transversal recursive least square algorithm (FTRLS), the linear random antenna are obtained by the use of the non uniform fast Fourier transform .This new adaptive beam-forming technique was compared with similar algorithms such as least mean square (LMS) and recursive least square (RLS) algorithms. Simulation results shows that this combination is better for beam-forming towards the desired user, also offers better interference suppression since it has the smallest minimum square error of about $1.0 * 10^{-2}$ compared to the least mean square algorithm that has minimum square error of $3.6^{*} 10^{-}$ ${ }^{2}$ and the recursive least square that gave minimum square error of $3.5 * 10^{-2}$, good tracking performance compared to other algorithms such as the least mean square (LMS) and recursive least square (RLS) algorithms since it produced the most narrow beam towards the signal of interest. It converges after 10 considering 1000 snapshots while the least mean square and the recursive least square algorithms converge after 40 and 50 considering 1000 snapshots respectively hence it have higher speed of convergence.
\end{abstract}

\section{General Terms}

Fast Recursive Least Square Algorithm, Random antenna array, Gaussian signal.

\section{Keywords}

Smart antenna system, Beam-forming, Fast Recursive Least Square Algorithm,

\section{INTRODUCTION}

The Wireless cellular communication has experienced a geometric growth in the demand for provision of wireless multimedia services such as internet access, multimedia data transfer, video calling or video conferencing [1-3].

A smart antenna is a unit of the wireless communication system and performs spatial signal processing with multiple antennas. Multiple antennas can be used at either the transmitter or the receiver. Recently, the technology has been extended to the use of multiple antennas at both the transmitter and receiver, such an antenna is called the multiple input multiple output (MIMO) system. As extending smart antennas technology, MIMO support spatial information processing in the sense that conventional research on smart antennas have focused on how to provide the beam forming advantage by the use of spatial signal processing and wireless channels. Spatial information processing includes spatial information coding, such as spatial multiplexing and diversity coding as well as beam forming.

In order to meet up with this up surge in demand and limited capacity that is offered by the conventional single input single output (SISO) systems, there is a need to use multiple inputs multiple outputs (MIMO) systems. The introduction of smart antenna systems provides greater capacity by reducing cochannel interferences and reducing the fading effects has been proposed [5-7].

Many studies have shown that when antenna array is appropriately used in a mobile communication system, it helps in improving the system performance by increasing channel capacity and spectrum efficiency, extending range coverage, tailoring beam shape and steering multiple beams to track mobiles. It also reduces multipath fading. The antenna array may be used with other methods such as channel coding, adaptive equalization and interference cancelling to enhance the system performance [15], [16]. However, these solutions are not satisfactory in practice; a promising approach to achieve these goals is the use of smart antennas at the base and mobile stations. In order to harness the full advantage of these systems, advanced space-time signal processing techniques need to be developed [7].

The previous research challenge encountered after the revolutionary advancement from the use of single antenna to multi-antenna array of smart antenna was to develop better antenna that can provide the expected efficient use of the limited spectrum, minimization of cost of establishing new wireless networks, which has been provided in smart antenna [4],[8].

Now, the recent research is to develop a new way in which the adaptive beam-forming algorithm can provide better interference mitigation of noise, adjacent channel interference, and multipath fading, faster speed of convergence.

In this paper, linear random antenna array with fast recursive least square is used to provide better interference mitigation, good tracking performance, and faster convergence.

\section{LITERATURE SURVEY}

Balasem [19] et al in the paper titled "Beam-forming Algorithms Techniques by using MVDR and LCMV" explains that a smart antenna system consist of an array of antennas that together directs different transmission or reception beam towards each user in the antenna system. This is called Beam-forming. Ali Hakeem [20] et al in the paper titled "Robust interference suppression using a New LMS Based Adaptive Beam-forming Algorithm" introduces a robust variable step size NLMS algorithm to improve interference mitigation in smart antenna system. Maialen [17] in the paper titled "Randomly Spaced Antenna Array" introduce randomly spaced antenna array with RLS algorithm to improve the interference suppression in smart antenna 
system.

\section{MATERIALS AND METHODS}

\subsection{Signal Model}

Within the framework of this paper, a randomly spaced linear antenna was considered for the main beam-forming model. Unlike regular smart array antennas in which the elements are spaced apart by a constant uniform value d, random array antennas are composed of randomly distributed elements [9], [10]. A random linear array (RLA)

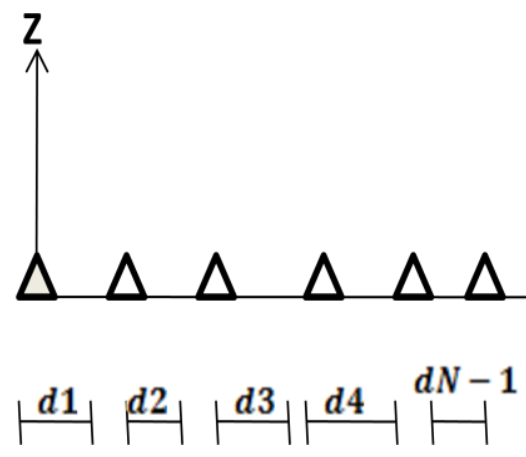

Figure 1: Random linear array

For RLA, the array was composed of $\mathrm{n}$ isotropic antenna elements, and $\mathrm{k}$ narrowband signals arriving from different directions with $\left(\left\{\theta_{0}, \phi 0\right\},\left\{\theta_{1}, \phi 1\right\}, \ldots,\left\{\theta_{k-1}, \phi_{k-1}\right\}\right)$ as the angles of elevation.

Due to the assumption that the incoming signals are located in the far-field and can be considered point sources, the arrival signals can be written as a uniform plane wave:

$$
\begin{aligned}
S_{i}(t)=S_{i t}(t) \cos & \left(\omega_{c} t\right) \\
& -S_{i Q}(t) \sin \left(\omega_{c} t\right) \\
& =R\left\{S_{i}(t) e^{j \omega_{c} t}\right\} .
\end{aligned}
$$

Where $s_{i t}(t)$ and $s_{i Q}(t)$ are phase and quadrature components respectively for each i-source,

$s_{i t}(t)=\sqrt{s_{i I}(t)^{2}+s_{i Q}(t)^{2}}$ is the envelope of the signal, and $\omega_{c}$ is the angular frequency of the signal.

Having a reference antenna, the signals arriving at the other antennas are related to the reference by a time delay:

For 1-dimension random linear array

$\tau_{1} D=\frac{d_{x} \sin \left(\theta_{i}\right)}{V_{o}}$

$\tau$ is the time delay for the elements.

$\mathrm{D}$ is the dimension

$\theta_{i}$ is the angle of arrival

$v_{o}$ is the speed of the signal

$S_{i t}(\mathrm{t})$, constitutes the signals from all directions including the desired signal

Where $d_{x}$ is the distance between the antenna elements. In general, the reference antenna is set at the origin to have zero phases.

As a consequence of this phase difference, the total signal arriving at the nth antenna element in snapshot $t$ is calculated as follows:

$X_{n}(t)=\sum_{i=0}^{k-1} s_{i}(t) e^{-j k_{i} \tau_{n}}+n(t)$
The steering vector is

$\mathrm{a}\left(\theta_{i}, \phi_{i}\right)=\left[1, e^{-\frac{2 \pi j}{\lambda_{i}} \tau_{1, \ldots . \prime}} e^{-\frac{2 \pi j}{\lambda_{i}} \tau_{N-1}}\right] . T$

Where $k_{i}=\frac{2 \pi}{\lambda_{i}}$ is the wave number vector, $\tau_{n}$ is the delay for the nth element that is the last element and $n(t)$ is the noise signal.

$\mathrm{A}$
$\left[\mathrm{a}\left(\theta_{o}, \phi_{o}\right), \mathrm{a}\left(\theta_{1}, \phi_{1}\right), \ldots, \mathrm{a}\left(\theta_{k-1}, \phi_{k-1}\right)\right]$

$(\theta, \phi)=$

The total signal can be expressed as a superposition of signals from all the sources by:

$\mathrm{X}(\mathrm{t})=\mathrm{A}(\theta, \phi) S_{i}(\mathrm{t})+\mathrm{n}(\mathrm{t})$

Where $\mathrm{X}(\mathrm{t})=\left[X_{1}(t), X_{1}(t), \ldots, X_{N}(t)\right]^{T}$ is the total baseband signal matrix and is the steering matrix that contains all the incoming sources. Considering all the snapshots, the total signal can be rewritten as follows:

$\mathrm{X}=\mathrm{A}(\theta, \phi) S_{i}(t) n(t)$.

Where

$\mathrm{X}=[\mathrm{x}(1), \mathrm{x}(2), \ldots, \mathrm{x}(\mathrm{L})]$.

$\mathrm{S}=[\mathrm{s}(1), \mathrm{s}(2), \ldots \ldots, \mathrm{s}(\mathrm{L})]$.

$\mathrm{N}=$

$[\mathrm{n}(1), \mathrm{n}(2), \ldots, \mathrm{n}(\mathrm{L})]$

\subsection{Fast Transversal Algorithm}

The fast transversal filter algorithm entails the combination of the use of four transversal filters for the forward and backward predictions, gain vector computation and joint process estimation. This algorithm has the advantage of reduced computational complexity.

Input parameters:

Tap-input vector $X_{N-1}(n-1)$, desired output $d(n)$

Tap weight vectors $\bar{a}_{N}(n-1), \bar{g}_{N}(N-1)$ and $\bar{w}_{N}(N-1)$

Normalized gain vector, $\bar{k}_{N}(n-1)$

$\mathrm{N}$ is the number of interferences to be considered

$\mathrm{n}$ is the number of antenna elements

\subsubsection{Forward prediction relations}

$$
\begin{aligned}
& \varepsilon_{f}(k, N)=x(k)-W_{f}{ }^{T}(K, N) x(k-1, N)(11) \\
& =X^{T}(k, N+1)\left[\begin{array}{c}
1 \\
-w_{f}(k, N)
\end{array}\right] \\
& e_{f}(k, N)=\frac{\varepsilon_{f}(k, N)}{\gamma(k-1, N)}[18] \\
& \xi_{f_{\text {min }}}^{d}(k, N)=\lambda \xi_{f_{\text {min }}^{d}}^{d}(k-1, N)+e_{f}(k, N) \\
& \gamma(k, N+1)=\frac{\lambda \xi_{f_{\text {min }}}^{d}(k-1, N)}{\xi_{f_{\text {min }}^{d}}(k, N)} \gamma(k-1, N) \quad(14) \\
& W_{f}(k, N)=w_{f}(k-1, N)+\phi(k-1, N) \varepsilon_{f}(k, N) \\
& x(k, N+1)=\left[x(k) x^{T}(k-1, N)\right]^{T} \\
& \varepsilon_{b}(k, N)=e_{b}(k, N) \gamma(k, N) \\
& \frac{\gamma(k, N+1)}{\gamma(k, N)}=\frac{\lambda \xi_{b_{\text {min }}}^{d}(k-1, N)}{\xi_{b_{\text {min }}^{d}}^{d}(k, N)}
\end{aligned}
$$


The result can be expressed as;

$$
\begin{aligned}
& \phi(k, N+1)= \\
& {\left[\begin{array}{c}
0 \\
\phi(k-1, N)
\end{array}\right]+\frac{1}{\xi_{f_{\text {min }}^{d}}^{d}(k, N)}\left[\begin{array}{c}
1 \\
-W_{f}(k, N)
\end{array}\right] \varepsilon_{f}(k, N)} \\
& \xi_{b_{\text {min }}}^{d}(k, N)=\lambda \xi_{b_{\text {min }}}^{d}(k-1, N)+\frac{\varepsilon b^{2}(k, N)}{\gamma(k, N)} \\
& W_{b}(k, N)=W_{b}(k-1, N)+\phi(k, N) e_{b}(k, N)
\end{aligned}
$$

$$
W_{f}(k, N)=w_{f}(k-1, N)+\phi(k-1, N) e_{f}(k, N)
$$

Where $\phi(k-1, N)=S_{D}(k-1, N) x(k-1, N)$

\subsubsection{Backward Prediction Relations}

$$
\begin{aligned}
& \varepsilon_{b}(k, N)=e_{b}(k, N) \gamma(k, N) \\
& \frac{\gamma(k, N+1)}{\gamma(k, N)}=\frac{\lambda \xi_{b_{\text {min }}}^{d}(k-1, N)}{\xi_{b_{\text {min }}}^{d}(k, N)} \\
& \xi_{b_{\text {min }}}^{d}(k, N)=\lambda \xi_{b_{\text {min }}}^{d}(k-1, N)+\frac{\varepsilon b^{2}(k, N)}{\gamma(k, N)} \\
& 1+\frac{\varepsilon b^{2}(k, N)}{\lambda \gamma(k, N) \xi_{b_{\text {min }}^{d}}^{d}(k-1, N)}=\frac{\varepsilon_{b_{\text {min }}}^{d}(k, N)}{\lambda \xi_{b \min }^{d}(k-1, N)} \\
& W_{b}(k, N)=W_{b}(k-1, N)+\phi(k, N) e_{b}(k, N) \\
& =W_{b}(k-1, N)+\phi(k, N) \varepsilon_{b}(k, N) \\
& {\left[\begin{array}{l}
\phi(k, N)]= \\
0
\end{array}\right]} \\
& \phi(k, N+1)-\frac{1}{\lambda \xi_{b_{\text {min }}}^{d}(k-1, N)}\left[-W_{b}(k-1, N)\right] e_{b}(k, N)(25) \\
& \phi_{N+1}(k, N+1)=\frac{e_{b}(k, N)}{\lambda \xi_{b_{\text {min }}}^{d}(k-1, N)}
\end{aligned}
$$

By applying equations (19), (25), and (20) in equation (21), we can show that;

$1+\phi_{N+1}(k, N+1) \varepsilon_{b}(k, N)=\frac{\gamma(k, N)}{\gamma(k, N+1)}$

$\gamma^{-1}(k, N)=\gamma^{-1}(k, N+1)-\phi_{N+1}(k, N+1) e_{b}(k, N)(28)$

\subsubsection{Joint-process estimation}

$e(k, N)=d(k)-W^{T}(k-1, N) x(k, N)$

$\varepsilon(k, N)=e(k, N) \gamma(k, N)$

$w(k, N)=w(k-1, N)+\phi(k, N) e(k, N)$

$W_{f}(-1, N)=W_{b}(-1, N)=W(-1, N)=0$

Vector $\phi(-1, N)$ set to zero assuming that the input and the desired signals are zero for $\mathrm{k}<0$, the conversion factor should be initialized

$\gamma(-1, N)=1[18]$

The computational complexity of the FTRLS algorithm is $7(N)+14$ multiplication per output sample. The key feature of the FTRLS algorithm is that it does not require matrix multiplications. Because of this, the implementation of the FTRLS algorithm has complexity of order N, multiplications per output sample.The forgetting factor is changed to 0.8 while other parameters remain the same. The step size is 0.001 , forgetting factor is 0.9 with the same 100 samples or iterations. Later, the step size is increased to 0.0001; forgetting factor is 0.9 while other parameters are maintained. The step size is then changed to 0.0001 ; forgetting factor is
0.9 with 1000 samples or iterations. The forgetting factor is changed to 0.99 with 1000 samples or iterations.Finally, the forgetting factor is varied to 0.8 with 1000 samples or iterations.Recently, Long Term Evolution (LTE) and WiMAX wireless communication technologies have been chosen as candidates for the so called $4 \mathrm{G}$ mobile communication systems. To achieve the required data rate and capacity, both WiMAX and LTE systems were introduced which support multi-antenna technologies including beam-forming, using up to 4 antenna elements for multi-input and multi-output (MIMO) enhancement strategy. Now, the advanced version of the LTE system called LTE advanced, array beam-forming techniques involving the use of 4 to 8 antenna elements in the base station was introduced to enhance the system performance. Thus, this requires the use of 8 -antennas elements for the new adaptive beam-forming technique for smart antenna system to meet the requirement of the data rate and capacity.The signal model was composed of $\mathrm{N}=8$ antenna elements randomly spaced along the $\mathrm{x}$-axis linear rectangular antenna (LRA) model and 3-narrow band signals, one desired and two interfering signals, arriving from $\theta_{\circ}=0^{\circ}, \theta_{1}=-30^{\circ}$ and $\theta_{2}=30^{\circ}$ at $2 \mathrm{GHz}$ frequency. The power of the incoming signals was $20 \mathrm{~dB}$ and $10 \mathrm{~dB}$ for the desired and interference signals respectively. The step size is 0.01 , forgetting factor is 0.9 with 100 samples or iterations.

\section{RESULTS}

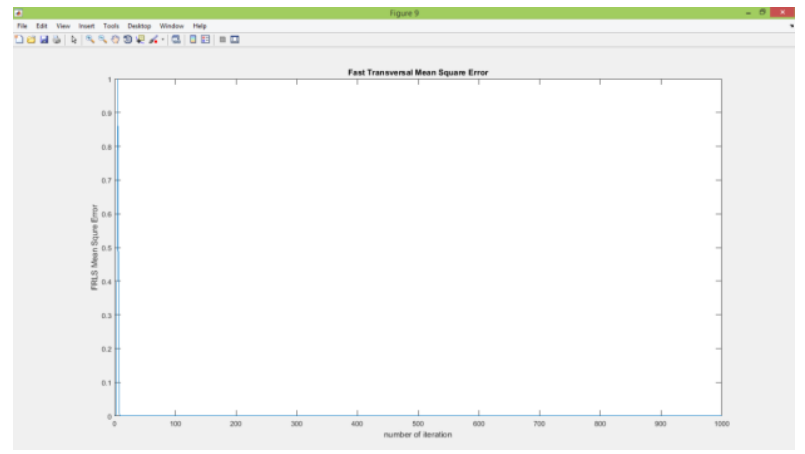

Fig 1: FTRLS Mean Square Error for the new FTRLS algorithm with forgetting factor of $0.99,8$-elements, 1000 iterations

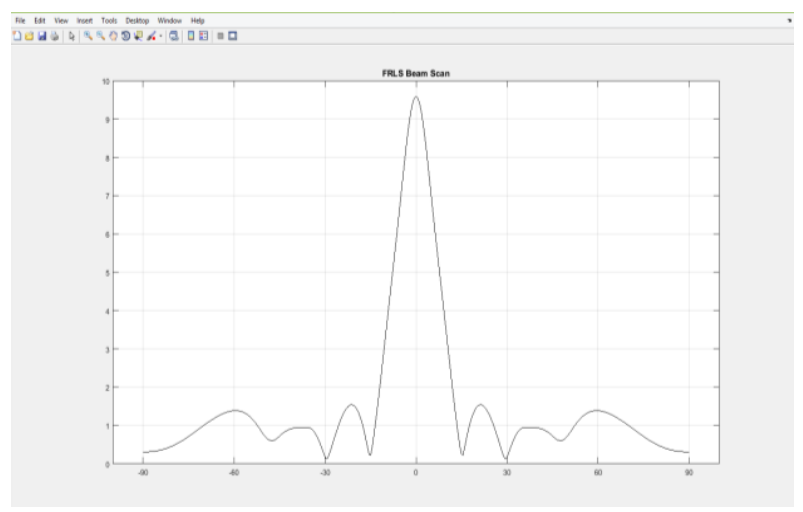

Figure 2: Beam-forming for FTRLS at iteration=100, number of elements $=8$, lambda $=0.99$ 


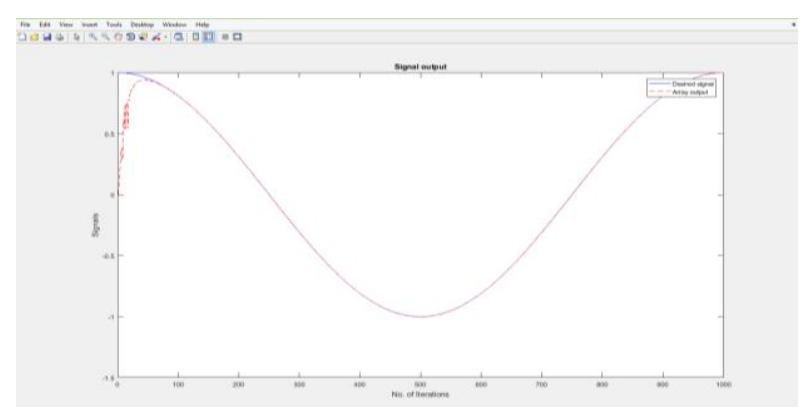

Fig 3: Tracking ability of the FTRLS algorithm

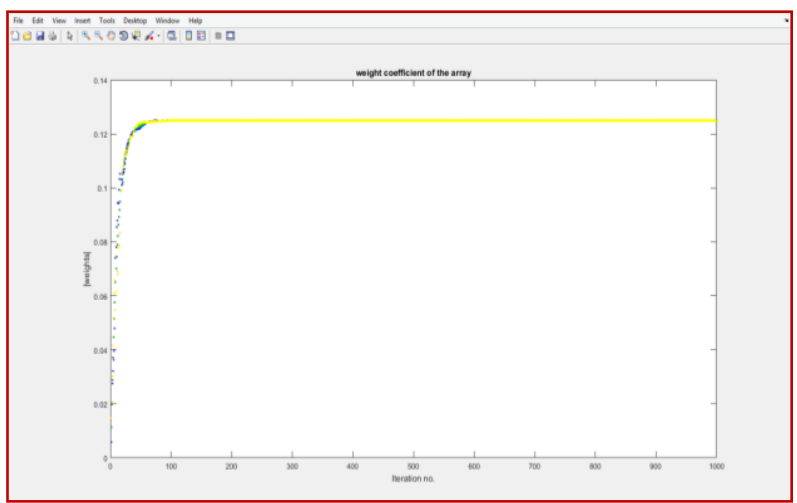

Fig 4: FTRLS Weight coefficients of the random antenna array

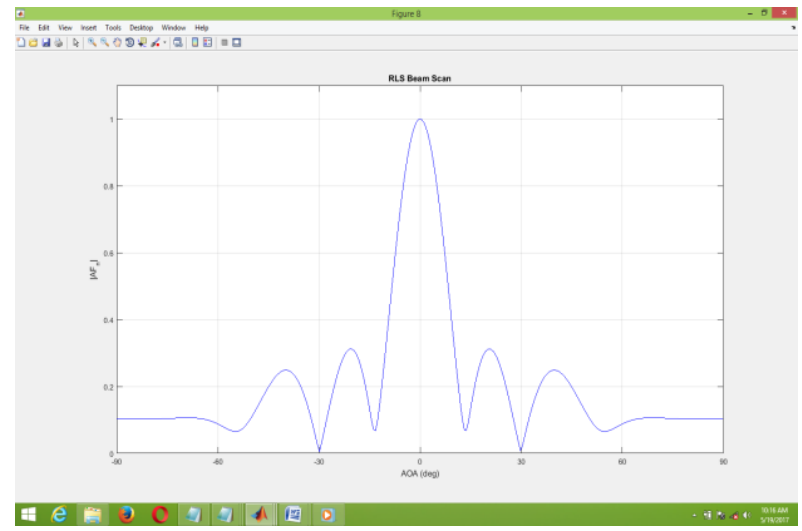

Figure 5: RLS Beam-forming, iterations $=100$, elements $=8, \lambda=0.99, \mu=0.001$,

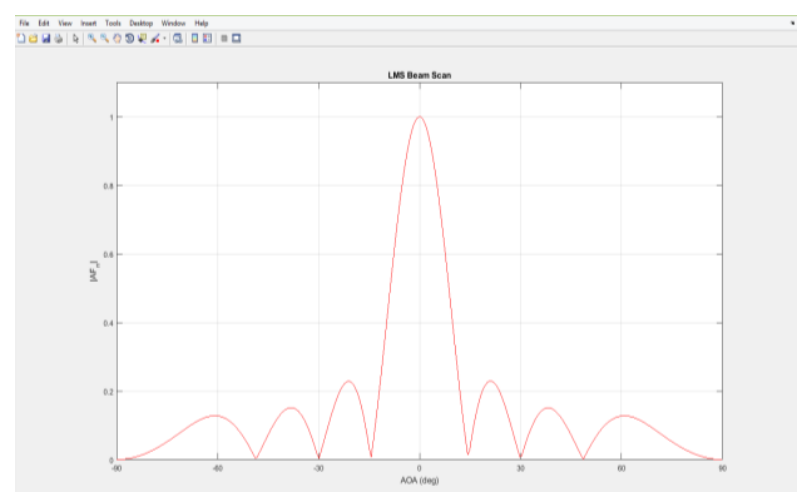

Figure 6: LMS Beam-forming at $\boldsymbol{\mu}=\mathbf{0 . 0 0 1}$, iterations $=100$, elements

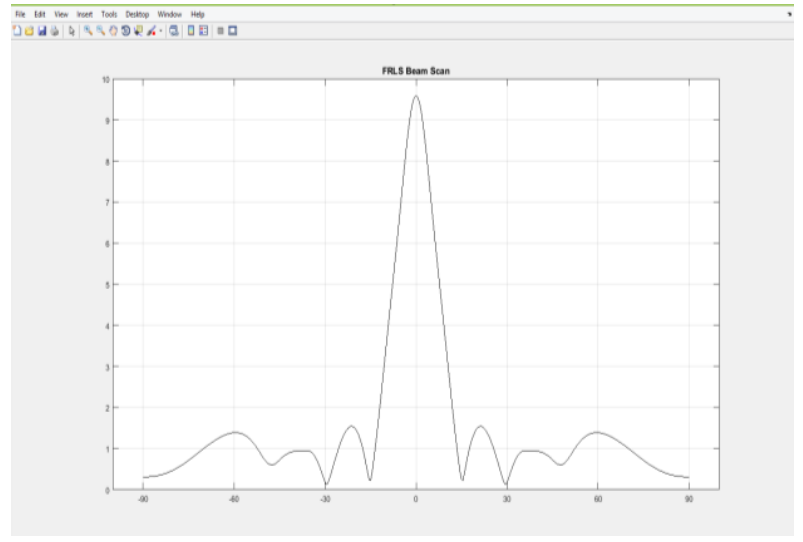

Figure 7: FTRLS Beam-forming, iterations=1000, elements $=8, \lambda=0.99$

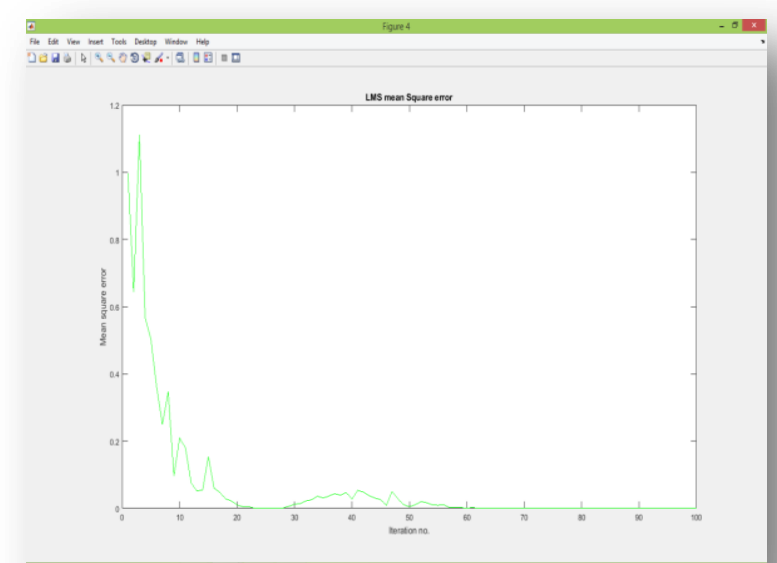

Figure 10: Mean square error for LMS algorithm, $\mu_{1}=0.01$ iterations $=100$

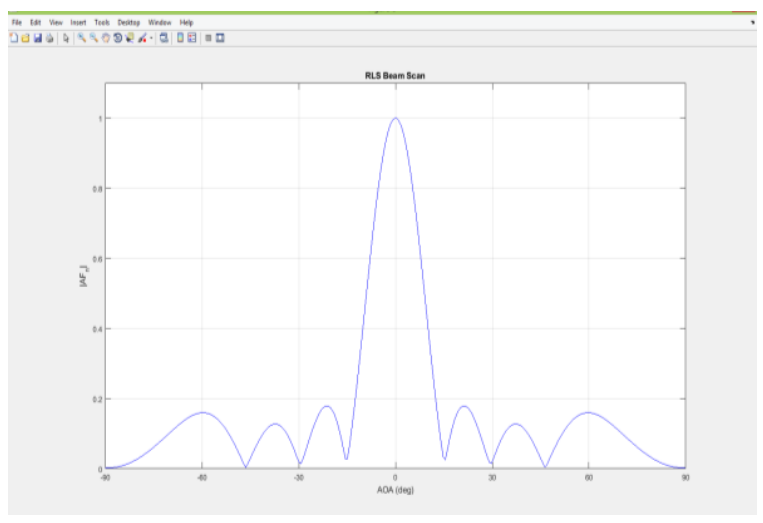

Figure 8: RLS Beam-forming at $\boldsymbol{\mu}=\mathbf{0 . 0 0 1}$, iterations $=1000$, elements $=8$ 


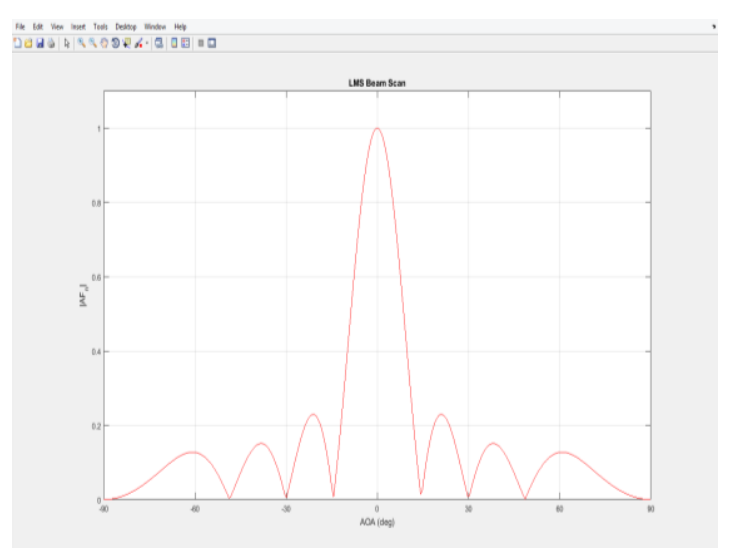

Figure 9: Beam-forming for LMS iterations=1000, $=0.0001$, number of elements $=8$

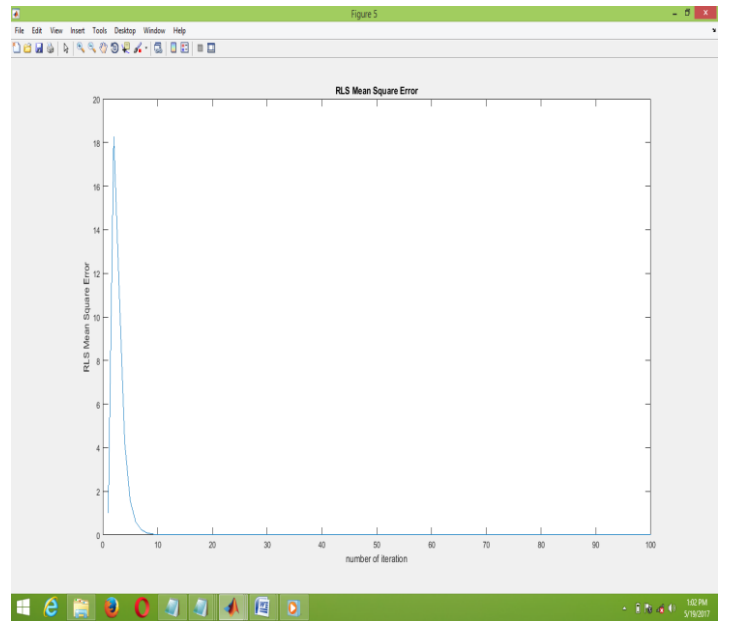

Figure 11: RLS Mean Square Error: $\lambda_{1}=0.9$, elements $=8$, iterations $=100$

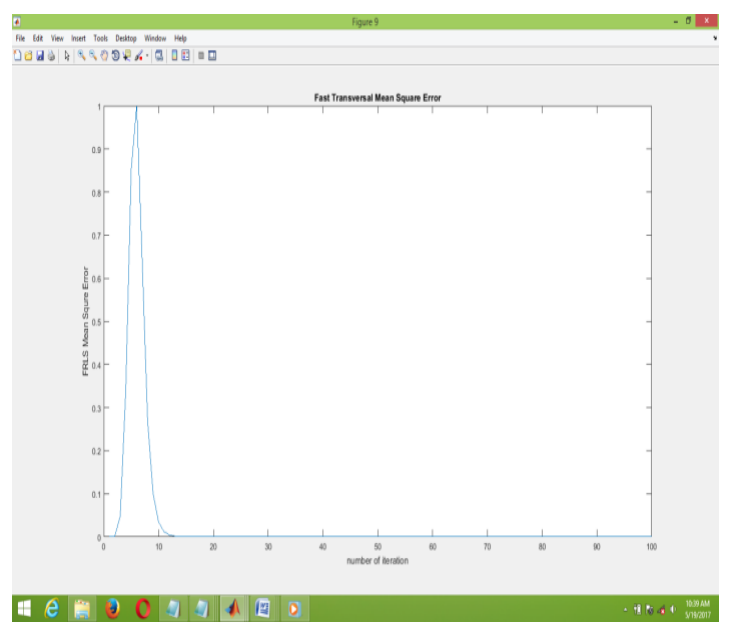

Figure 12: The FTRLS Mean square error at $\lambda=$ 0.99 , iterations $=100$, elements $=8$

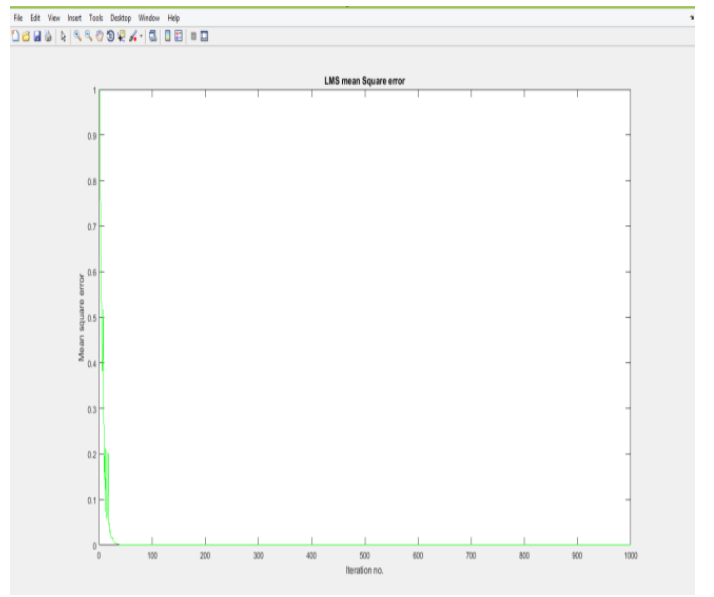

Figure 13: LMS Mean Square Error at iteration=1000, element $=8, \mu=0.0001$

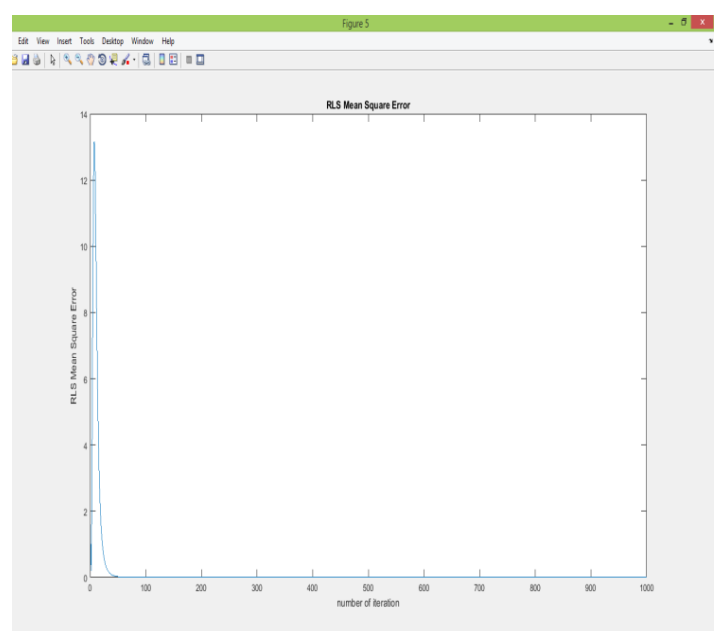

Figure 14: RLS Mean Square Error at iterations=1000, elements $=8, \lambda=0.99$

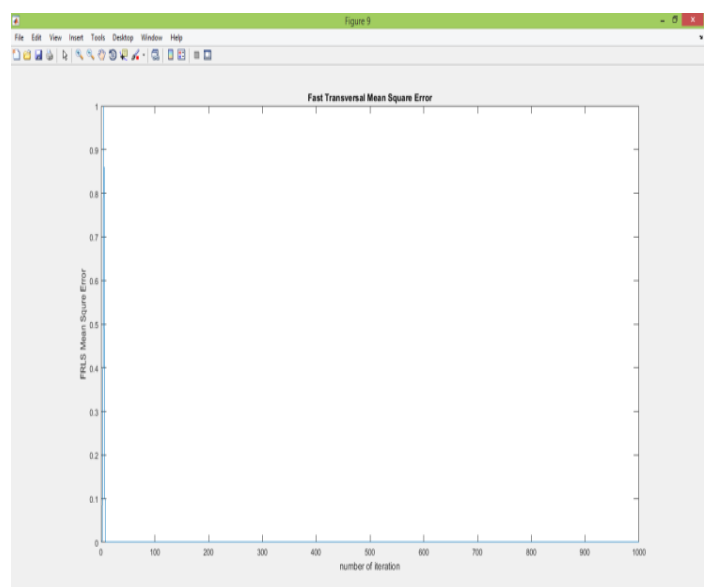

Figure 15: FTRLS Mean Square Error at iterations=1000, elements $=8, \lambda=0.99$ 
Table 1: Results of the simulation interpretation

\begin{tabular}{|c|c|c|c|c|c|c|c|}
\hline Parameters & Step & $\begin{array}{r}\text { Number } \\
\text { size }\end{array}$ & $\begin{array}{l}\text { Forgetting } \\
\text { of elements }\end{array}$ & $\begin{array}{l}\text { Number } \\
\text { factors }\end{array}$ & $\begin{array}{r}\text { Signal } \\
\text { of iterations }\end{array}$ & $\begin{array}{l}\text { MSE Conv } \\
\text { frequency }\end{array}$ & rgence \\
\hline \multirow[t]{2}{*}{ LMS } & 0.01 & 8 & - & 100 & $2 \mathrm{GHz}$ & $3.6 * 10^{-2}$ & 60 \\
\hline & 0.0001 & 8 & - & 1000 & $2 \mathrm{GHz}$ & & 40 \\
\hline \multirow[t]{2}{*}{ RLS } & -- & 8 & 0.8 & 100 & $2 \mathrm{GHz}$ & $3.5^{*} 10^{-2}$ & 9 \\
\hline & & 8 & 0.99 & 1000 & $2 \mathrm{GHz}$ & & 50 \\
\hline \multirow{2}{*}{\multicolumn{2}{|c|}{ FTRLS -- }} & 8 & 0.8 & 100 & $2 \mathrm{GHz}$ & $1.0 * 10^{-2}$ & 8 \\
\hline & & 8 & 0.99 & 1000 & $2 \mathrm{GHz}$ & & 10 \\
\hline
\end{tabular}

Table 2: Interpretation of the simulation

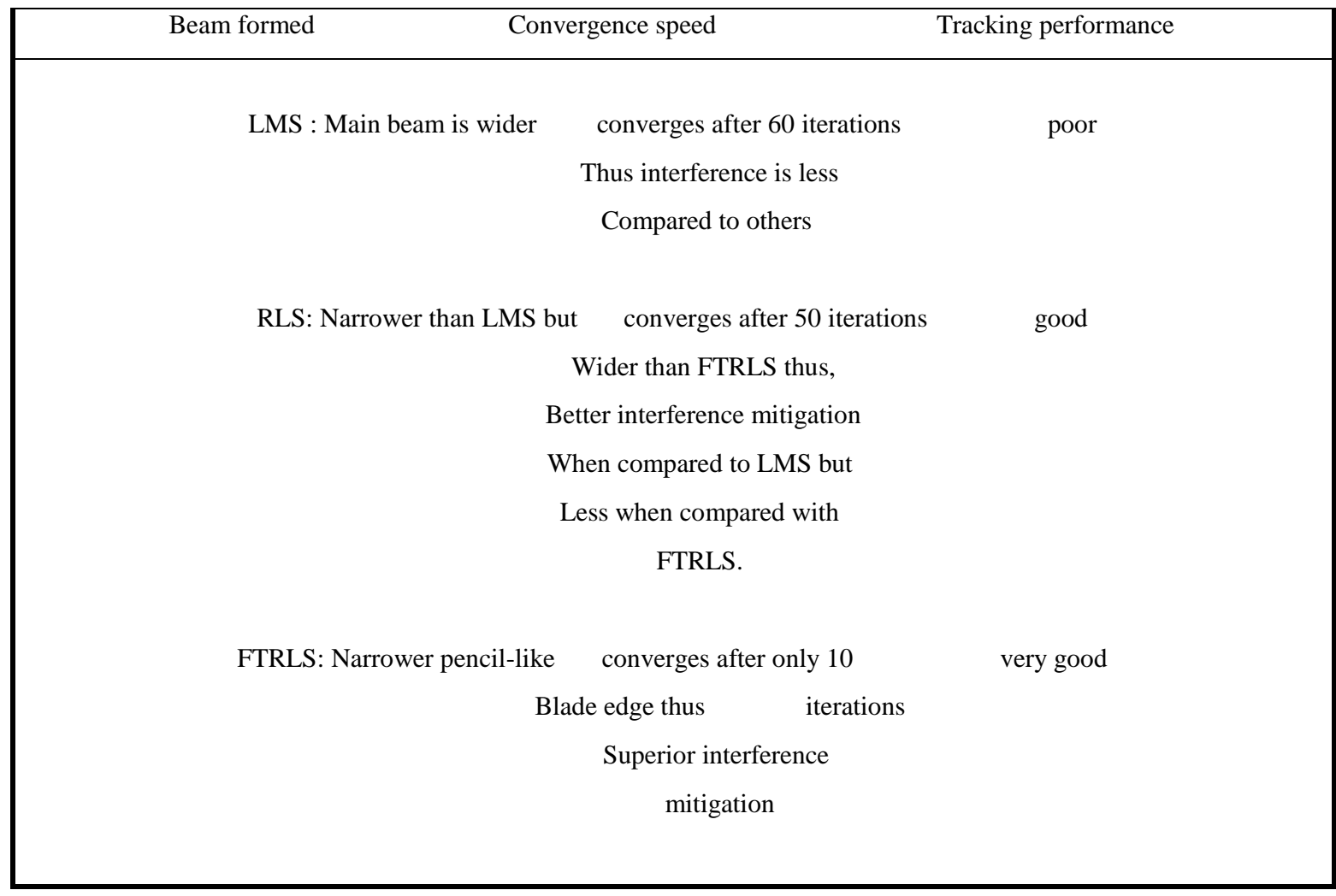




\section{DISCUSSION AND CONCLUSION}

From the findings, one can see that the MSE of the new Algorithm technique converges only after 8 iterations (for a total of 100 iterations) and for a total of 1000 iterations it converges after only 100 iterations also, it has better tracking performance which is depicted after only 40 iterations compared to the LMS and RLS algorithms which converges after 60 iterations (for a total of 100 iterations And converges after 40 iterations (for a total length of 1000 iterations) while RLS converged after 9 iterations (for 100 iterations)and after 50 iterations (for total of 1000 iterations). Hence, this have shown that the new algorithm technique that is the FTRLS algorithm in a randomly spaced antenna configuration provided faster convergence rate, better interference mitigation over the LMS algorithm and RLS algorithm.

\section{ACKNOWLEDGEMENT}

Thanks to my co-authors who contributed to the success of this research work.

\section{REFERENCES}

[1] Ueng F.B. Smart Antenna for multiuser DS/CDMA Communication in multipath fading channels, IEICE Trans on Communications. 2005. 88: pp 2944-2954.

[2] Bellofiore S. Smart Antenna System For Mobile Communication networks, Beam-Forming and Networks throughput, IEEE Antenna and Propagation Magazine. 2002 . 44: pp 106-114.

[3] Boukalov, A. O. and Haggman S.G., System Aspects of System Antenna Technology in Cellular Wireless Communication. IEEE Trans on Microwave Theory and Techniques. 2000. 48(1): 919-929.

[4] Balanis C. Antenna Theory Analysis and Designs, 3rd ed. New York: Hoboken, N. J Wiley; 2005.

[5] Arunitha .A. Gunasekaran, A., Senthil, T, and Senthilvel, N. Adaptive Beam-Forming Algorithms for MIMO Antenna. International Innovation Technology and Exploring Engineering Journal (IJITEE) .2015 4th ed. pp 9-12.

[6] Qinghua, L., Guangije, L., Wookbon, L.,Moon-li, L., David, M. Brono,C. and Zexian, L..MIMO techniques in WiMAX and LTE: a feature overview IEEE Communications Magazine, 2010. 48(1):8692.

[7] Thamer .M.J. Performance Enhancement of Smart Antennas Algorithms for Mobile s and Signal Processing Communication Systems .International Journal of Circuits, System. 2014 32: pp. 313-320.

[8] Allen. B. and Ghavani. M. Adaptive Array Systems Fundamentals and Applications. London, UK. John Wiley and Sons. 2005
[9] Zhenwer. L. Antenna Array Pattern Null Steering, China; 1984. pp 2304-2345.

[10] Stutzman, W. L. Shaped-beam synthesis of nonuniformly spaced linear arrays, IEEE Trans. on Acoustic, Speech and Signal processing, 1972. 20, pp. 499-501.

[11] Slock D.T. and KAilath. T. Numerically Stable Fast Transversal Filters For Recursive Least Squares Adaptive Filtering. IEEE Trans on Signal Processing; 1993.32: pp 92-113.

[12] Cioffi .M. and Kailath.T. Fast Recursive Least Squares, Transversal Filters for Adaptive Filtering. IEEE Trans on Signal Processing; 1984. 32: pp 304-337.

[13] Eweda. E. Comparison of RLS, LMS and Sign Algorithms for Tracking Randomly Time Varying Channels. IEEE Trans on Signal Processing, 2014. $42^{\text {nd }}$ 11: pp 2937-2944.

[14] Usha M. Nalini, K.Ganesh, P. Raghavendra, V. Habib, U.K. Laksmi, D.R. and Madhav, B. Non-Blind Adaptive Beam Forming Algorithms for Smart Antennas. International Journal of recent Research and Applied Studies. IJRRAS, 2011. $6^{\text {th }}$ edition, 4: pp 491-495.

[15] Mouhamadou.M and Vaudon .P . Smart Antenna Array Patterns Synthesis: Null Steering and Multi-user BeamForming by Phase Control. Progress in Electromagnetic Research, PIER, 2006. .60: PP. 95-106.

[16] Liu, Q.H. and Nguyen, N. An accurate algorithm for non-uniform fast Fourier transforms (NUFFT's), IEEE Microwave Guided Wavelet, 1998. 8, pp. 18-20

[17] Maialen, C.V. Randomly Spaced Smart Antennas. University of New Mexico, Mexico. MSc, thesis. Department of Electrical Engineering, University of New Mexico, Mexico, 2010, pp. 85.

[18] [18] Jalal, A. S, Kah-seng, C. and Ali, M. Adaptive Array Beam-forming Using a Combined LMS-LMS Algorithm IEEE Transactions on Antennas and Propagation, 2010. 58, pp 3545-3557.

[19] Balasem. S., Tiong, S.K. and Koh, S. Beam-forming Algorithms Techniques by Using MVDR and LCMV.World Applied Programming. 2012. $5^{\text {th }}$ edition, 2: $315-324$

[20] Ali, H. Raed, S., Shihab, J. and Ehab, S. Robust Interference Suppression Using a New LMS Based Adaptive Beam-forming Algorithm 17th IEEE Mediterranean Electro-technical Conference, Beirut, Lebanon. 2014, pp. 13-16. 